\title{
Study on the Motion state of Powdery materials in Dense-phase Pneumatic Conveying Pipe
}

\author{
Zhihua Li ${ }^{\mathrm{a}}$, Guangpeng Liu , Nan Guo and Feipeng Liu \\ College of Mechanical and Electrical Engineering, Qingdao University of Science \& Technology, Qingdao 266061, China
}

\begin{abstract}
Using the method of computational fluid dynamics, a model is created about powdery materials flowing in the dense-phase pneumatic conveying pipe. the motion state powdery materials flowing in the horizontal and vertical pipe is simulated. It is found that in the horizontal pipe the powdery materials represent the flow of dune-like state, and continuously move forward in this form, the volume fraction of powdery materials at the pipe's bottom is large, the velocity is low; In the vertical pipe, the columnar solid-plug can form and scatter continuously, and the motion state is closer to fluidization.
\end{abstract}

\section{Introduction}

Pneumatic conveying technology is using compressed gas as conveying power in a sealed pipe to convey granular materials. It is widely used to convey powdery materials such as powdery materials in rubber industry [1]. Pneumatic conveying technology has been developed from the dilute-phase conveying to the dense-phase conveying. Compared with the dilute-phase conveying, the dense-phase conveying has many advantages such as less energy consumption, large conveying capacity and low breakage rate, but there are some problems such as complex flow form, poor conveying stability and high blockage rate of conveying pipe [2]. In order to provide more precise and powerful references for the research and design of the dense-phase pneumatic conveying system, it is necessary to carry out numerical simulation and analysis of motion state of powdery materials in the horizontal and vertical conveying pipe[3].

\section{The theoretical basis and establishment of mathematical model}

The Fluent software in the CFD (Computational Fluid Dynamics) software package is used as the platform to simulate the motion state of powdery materials in dense-phase pneumatic conveying pipe. The problem of multiphase flow usually has two solving methods: the Euler-Euler method and the Euler-Lagrange method. The Euler-Euler method is to solve the N-S (Navier-Stokes) equation for the continuous phase fluid in the Eulerian framework, and to solve the particle phase conservation equation for the particle phase in the Eulerian framework; The Euler-Lagrange method is to solve the N-S (Navier-Stokes) equation for the continuous phase fluid in the Eulerian framework, but to solve the particle trajectory equation for the particle phase in the Lagrange framework.

\subsection{The theoretical basis}

The Euler-Euler model is used to calculate the gas-solid phase flow. Its basic idea is to treat the particles as pseudo fluid. The model has its own convection term, diffusion term, production term and dissipation term. In the Fluent software, there are three kinds of Euler-Euler multiphase flow model, which are VOF model, Mixture model and Eulerian model. The Eulerian model is available to simulate the particle motion state for the dense-phase pneumatic conveying of powdery materials.

Eulerian model can solve the momentum equation and continuity equation for each phase, realize coupling through pressure and interphase exchange coefficient, and obtain the flow characteristics of particles through using the molecular motion theory for granular flow. For dense-phase pneumatic conveying, the coupling between particles and gas phase is two-way, and there is a coupling between particle pressure and particle viscous stress caused by the collision between particles. The basic control equations of Eulerian model are as follows.

Continuous equation:

$$
\frac{\partial \rho}{\partial t}+\nabla \cdot(\rho \vec{v})=0
$$

Momentum equation:

$$
\frac{\partial(\rho \vec{v})}{\partial t}+\nabla \cdot(\rho \overrightarrow{v v})=-\nabla \cdot p+\nabla \cdot \vec{\tau}+\vec{F}
$$

\footnotetext{
${ }^{\text {a }}$ Corresponding author: lizh@mesnac.com

(C) The Authors, published by EDP Sciences. This is an open access article distributed under the terms of the Creative Commons Attribution License 4.0 (http://creativecommons.org/licenses/by/4.0/).
} 
Where, $\rho$ is the density of powdery materials, $\mathrm{kg} / \mathrm{m}^{3} ; t$ is the conveying time, $\mathrm{s} ; \vec{v}$ is the conveying velocity vector of materials, $\mathrm{m} / \mathrm{s} ; p$ is the pressure on micro unit of fluid, $\mathrm{Pa} ; \vec{F}$ is the external body force on the micro unit, $\mathrm{N} / \mathrm{m}^{3} ; \vec{\tau}$ is the viscous stress on the surface of micro unit, $\mathrm{Pa}$.

\subsection{The mathematical model}

The model is established in the pre-processing software named Gambit. The diameter of the pneumatic conveying pipe is $150 \mathrm{~mm}$. The velocity-inlet boundary condition is used as the powdery material (carbon black) inlet boundary and gas inlet boundary, and the pressure-outlet boundary condition is used as the outlet boundary. Because the pneumatic conveying pipe is slender, and the length of computational domain size is large, the author intends to use the two-dimensional double-precision solver. The standard $\kappa-\varepsilon$ double equation turbulence model is used as the turbulence model. The pressure coupling equations are solved by SIMPLE (Semi-Implicit Method for Pressure Linked Equations, the half implicit method of the pressure coupling equations), which is widely used for the calculation of flow field in computational fluid mechanics. The second-order upwind is used for the difference scheme.

\section{Simulation and analysis}

\subsection{The parameters of dense-phase pneumatic conveying system}

Table 1. shows the parameters of pneumatic conveying system in the simulation process.

Table 1. The parameters of dense-phase pneumatic conveying system

\begin{tabular}{|c|c|}
\hline Parameters of the system & The value of parameters \\
\hline $\begin{array}{l}\text { The stacking density of carbon } \\
\text { black }\left[\mathrm{kg} / \mathrm{m}^{3}\right]\end{array}$ & 375 \\
\hline The conveying capacity $[\mathrm{t} / \mathrm{h}]$ & 8 \\
\hline $\begin{array}{c}\text { The conveying gas } \\
\text { volume }\left[\mathrm{m}^{3} / \mathrm{h}\right]\end{array}$ & 280 \\
\hline $\begin{array}{l}\text { The conveying gas } \\
\text { velocity }[\mathrm{m} / \mathrm{s}]\end{array}$ & 10 \\
\hline Solid-gas ratio & 22 \\
\hline $\begin{array}{l}\text { The diameter of conveying } \\
\text { pipe }[\mathrm{mm}]\end{array}$ & 150 \\
\hline $\begin{array}{l}\text { The distance of horizontal } \\
\text { conveying }[\mathrm{m}]\end{array}$ & 3 \\
\hline $\begin{array}{l}\text { The distance of vertical } \\
\text { conveying }[\mathrm{m}]\end{array}$ & 15 \\
\hline
\end{tabular}

The phase diagram is a set of curves to describe the relationship between superficial gas velocity ug and unit length of pipe pressure drop $\Delta \mathrm{p}_{\mathrm{L}}$ through the solid mass flow $\mathrm{q}_{\mathrm{m}}$. It can accurately describe the flow characteristics of pneumatic conveying, and intuitively present the relationship between pressure loss and superficial gas velocity. It also can be used on the research of flow form division, stability analysis and optimization of parameters [4]. The typical phase diagram of Zenz in horizontal pipe is shown in figure 1.

In figure 1, when the superficial gas velocity ug at point $\mathrm{A}$ and larger, the flow form is dilute phase suspension flow. The influence of materials gravity is small, and the suspension force acted on powdery materials mainly comes from the vertical velocity component of turbulent gas flow and the bounce force when powdery materials collide with pipe wall. When the superficial gas velocity reduces to point $\mathrm{B}$, a portion of particles begin to settle down from the suspension flow. And there is a concentration gradient of powdery materials in the pipe cross section, which forms the stratified flow. When the superficial gas velocity continues reducing to point $\mathrm{C}$, the solid phase starts to separate from gas phase, and particles start to slide along the bottom of the pipe at a low speed in the role of air drag force and collision, which forms the moving bed. Curve CEF represents the connection line of the minimum pressure drop, as well as the dividing line of stable flow and unstable flow. Continue to reduce the superficial gas velocity to point $\mathrm{D}$, and the powdery materials move forward continuously in dune-like state with the accumulation of particles. The powdery materials that come into the pipe later repeat the process of settlement, showing the next dune-like state. So the forward motion state of a section of dune-like materials has formed in the whole horizontal pipe. At present, the particle-particle interactions and the interaction of particles and pipe wall have the biggest effect on the motion state. The simulation about the motion state of powdery materials in horizontal conveying pipe at different times is shown in figure 2 .

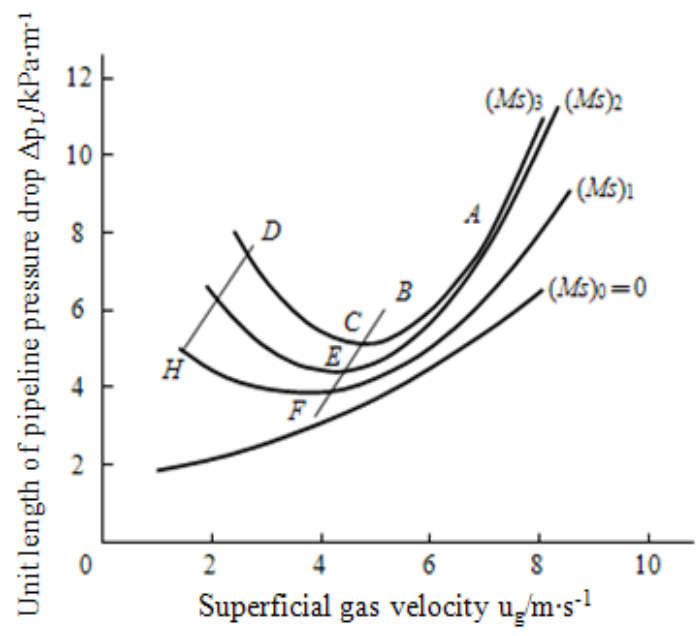

Figure 1. The typical phase diagram of Zenz in horizontal pipe

\subsection{The horizontal conveying pipe}




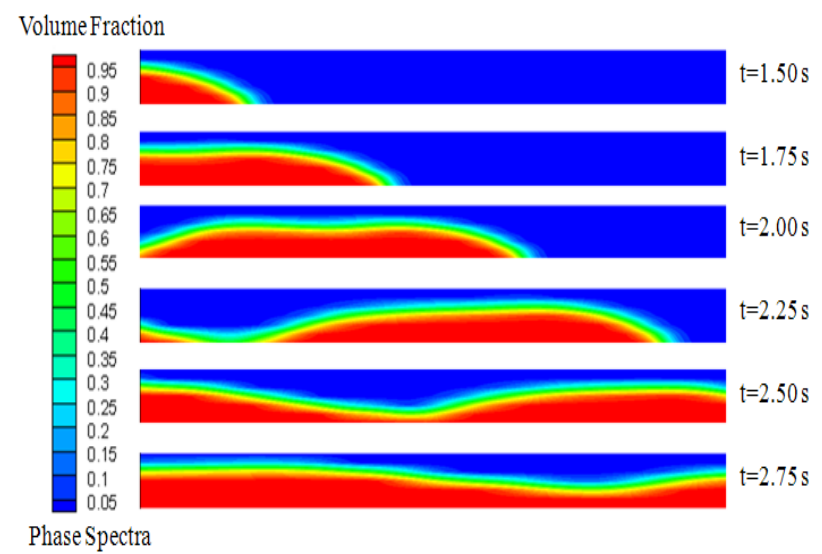

Figure 2.The motion state of the powdery material in horizontal conveying pipe at different times

Figure 3. and figure 4. respectively show the volume fraction of the powdery material and the distribution of velocity along the pipe diameter (Y-axis) in a certain cross section of the horizontal conveying pipe. In the figures, $\mathrm{Y}=0 \mathrm{~m}$ represents the bottom of the pipe and $\mathrm{Y}=$ $0.15 \mathrm{~m}$ represents the top of the pipe.

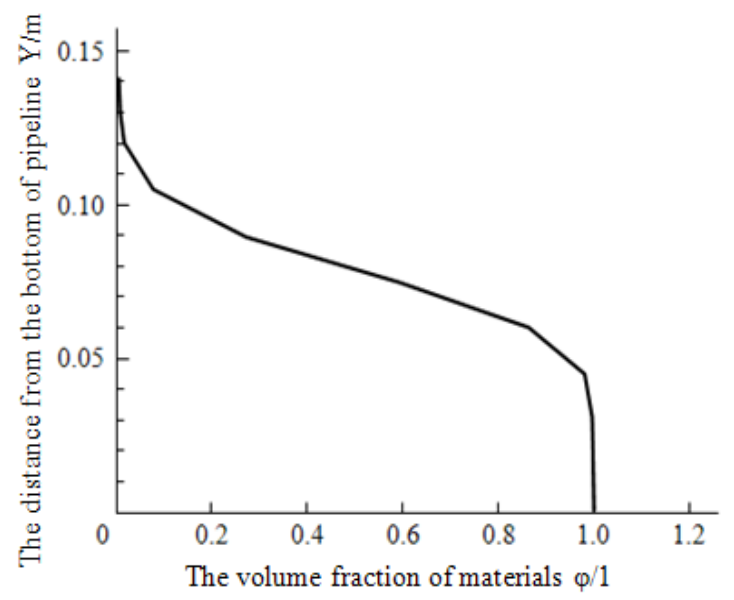

Figure 3. The volume fraction of the powdery material changes along the pipe diameter direction

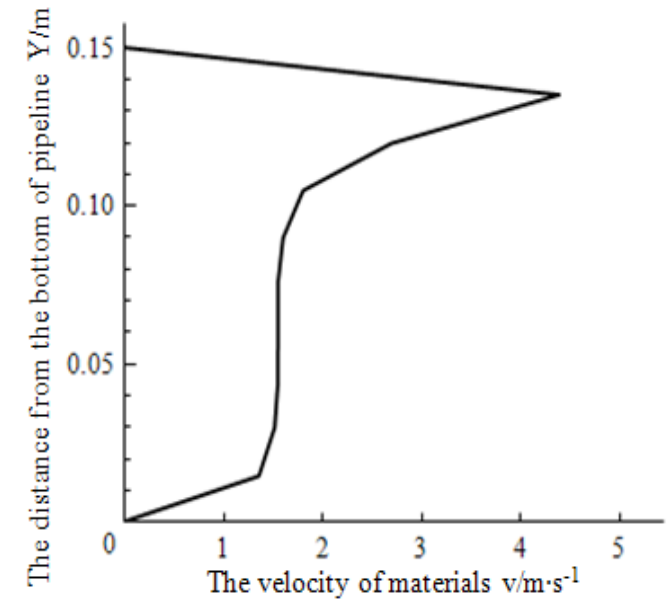

Figure 4. The velocity of the powdery material changes along the pipe diameter direction
The comprehensive analysis about figure 2., figure 3. and figure 4 . shows that the powdery material particles mostly concentrate in the bottom of the horizontal conveying pipe, the void ratio of the powdery material is small, and the velocity of the powdery material is relatively low. The volume fraction of the powdery material at the top of the horizontal pipe is small, the void ratio of the powdery material is high, and the velocity of the powdery material is relatively high. Solid-plug moves forward in the form of wave, along with the displacement movement of particles in solid-plug and accumulation layer. The velocity of the powdery material in the pipe wall is zero, the more close to the pipe wall, the greater velocity gradient of the powdery material will be. In figure 3, the accumulation layer thickness of the powdery material can be determined by the height of curve that the volume fraction of the powdery material is close to 1 . Accumulation layer thickness will decrease with the increase of superficial velocity. The velocity of the powdery material in the accumulation layer is very low, and it moves forward with the entrainment effect caused by the movement of the dune-like solid-plug. The thickness of accumulation layer changes along with the conveying distance, the more close to the end of the conveying pipe, the smaller accumulation layer thickness will be. The simulation results are consistent with the experimental results [5].

\subsection{The vertical conveying pipe}

The relationship between unit length of pipe pressure drop $\Delta \mathrm{p}_{\mathrm{L}}$ and superficial gas velocity ug in the vertical conveying pipe for different conveying capacity is shown in figure 5 [6].

As figure 5 shows, the relationship about the volume flow of powdery materials is $\mathrm{Q}^{0}<\mathrm{Q}^{1}<\mathrm{Q}^{2}<\mathrm{Q}^{3}$. Segment $\mathrm{AB}$ represents the relationship between unit length of pipe pressure drop $\Delta \mathrm{pL}$ and superficial gas velocity ug under the condition that the volume flow of powdery materials $\mathrm{Q}^{0}=0$. Taking curve $\mathrm{Q}^{1}$ as an example, when the superficial velocity is high, the volume fraction of powdery materials is relatively low. In this case, the powdery material is in dispersed state, that is, the dilute phase conveying. In the CD stage, with the reduction of superficial gas velocity, the solid-gas mixture ratio increases gradually, the material gravity pressure drop $\Delta \mathrm{p}_{\mathrm{g}}$ increases, but the overall pressure drop $\Delta \mathrm{p}$ decreases. This shows that the pressure drop $\Delta \mathrm{p}_{\mathrm{f}}$ generated by pipe wall friction reduces much larger than the increase in the pressure drop $\Delta \mathrm{p}_{\mathrm{g}}$ generated by the powdery material gravity. In the DE stage, with the continuous reduction of superficial gas velocity, the overall pressure drop $\Delta p$ decreases gradually and reaches the lowest values, but the proportion of the pressure drop $\Delta \mathrm{p}_{\mathrm{g}}$ generated by the powdery material gravity is large. In the EF stage, with the further reduction of superficial gas velocity, the solid-gas mixture ratio increases further, the pressure drop $\Delta \mathrm{p}_{\mathrm{g}}$ generated by the powdery material gravity plays a dominant role in the pipe. The overall pressure drop $\Delta \mathrm{p}$ increases, and the volume fraction of powdery materials 
in the pipe is relatively high. In this case, the powdery material is in aggregation state, that is, the dense phase conveying. When the superficial gas velocity is lower than its value in point $F$, the relative velocity between gas and particles cannot make the powdery material remains in suspension, which will produce a packed bed flow or moving bed flow.

The simulation about the motion state of the powdery material in vertical conveying pipe at different times in the EF stage is shown in figure 6. Because of the adhesion among material particles, the particles gather together and move upward in the role of suspension force generated by the upward flow of gas. When the solid-plug moves upward, it undertakes the falling materials from the previous solid-plug, and scatters corresponding amount of materials at the end of the solid-plug. So the solid-plug bed is similar to the fluidized state. The simulation results are consistent with the experimental results [5].

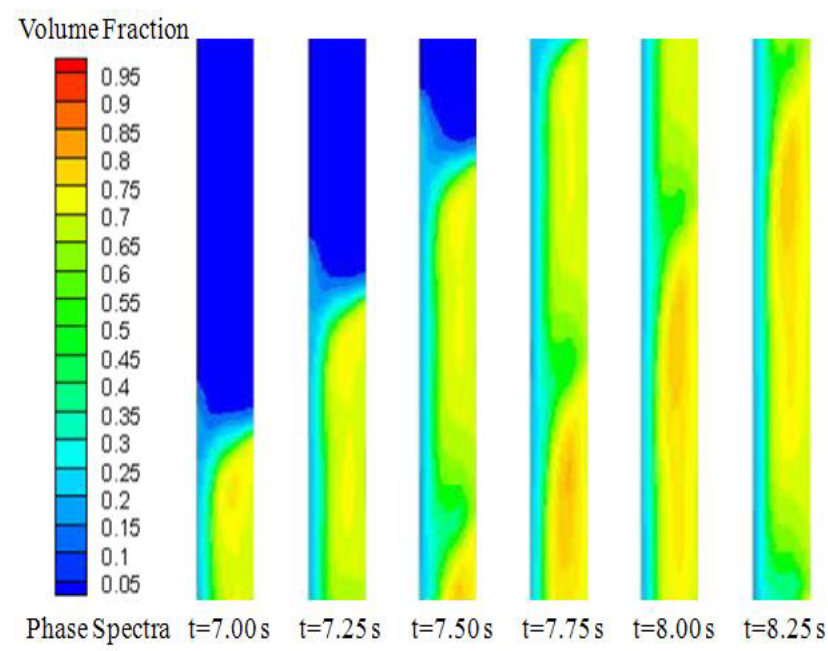

Figure 5. The relationship between unit length of pipe pressure drop and superficial gas

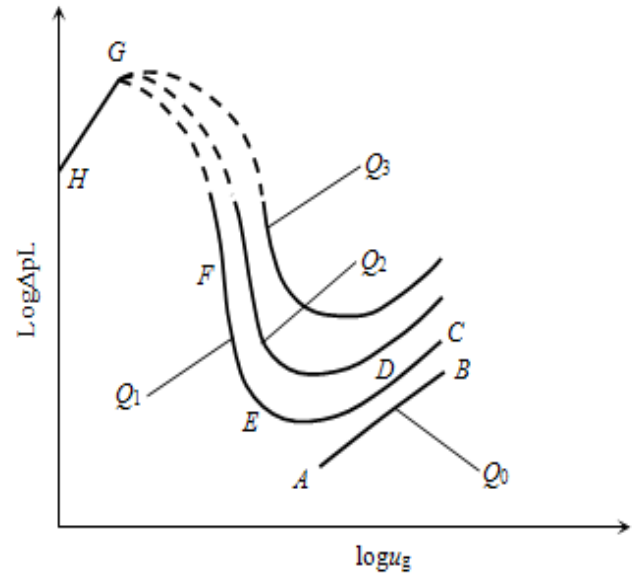

Figure 6 .The motion state of materials in vertical conveying pipe at different timesvelocity in vertical pipe
The following conclusions can be obtained by using Fluent software to simulate the flow process of powdery materials in dense-phase pneumatic conveying horizontal and vertical pipes.

(1) In horizontal pipe conveying process, powdery materials can represent the flow of dune-like state, and continuously move forward in this form. Powdery materials which are deposited at the bottom of pipe can form the accumulation layer. The larger the superficial gas velocity is, the smaller the thickness of accumulation layer will be. The volume fraction of powdery materials at the bottom of pipe is large, so the velocity is low, and the volume fraction of powdery materials at the top of pipe is small, so the velocity is high.

(2) In vertical pipe conveying process, because of the two-way role of suspension force generated by the upward flow of gas and the gravity of powdery materials, the columnar solid-plug can form and scatter continuously, showing the state of fluidization.

(3) The simulation results are consistent with the actual pneumatic conveying experimental results. This shows that the numerical simulation method can be used to research the dense-phase pneumatic conveying mechanism and the optimization design of pneumatic conveying system.

\section{References}

1. S.L.Chen,X.D.Yang, Q. Shi, Traffic information and security,30,74 (2012)

2. L.Yang,Y.H.Xie,Pneumatic Conveying
Engineering,
(China Machine

Press, Beijing,2006)

3. Z.H. Li,X.C. Li,G.P. Liu, Rubber Industry,62,496 (2015)

4. Q.M. Meng, Y. Zhou, X.P. Chen, et al, Boiler Technology, $42,4(2011)$

5. Z.H. Li, Y.Q.Liu ,P. Xia, Hoisting and Conveying Machinery, 87,75 (2009)

6. W.X. Peng, Y.Q. Wu , Pneumatic Conveying and Transporting in Workshop (China Measure Press, Beijing,2009)

\section{Conclusion}

